\title{
Mechanisms of up-conversion excitation of blue emission in YAG:Tm, Yb
}

\author{
A. KNÜPFER, V. OSTROUMOV, E. HEUMANN, G. HUBER and V. LUPEI*
}

Universität Hamburg, Institut für Laser-Physik, Jungiusstrasse 11, 20355 Hamburg, Germany

* Institute of Atomic Physics, Bucharest 76900, Romania

\begin{abstract}
$\underline{\text { Abstract }}$
Trivalent ytterbium ions were used as sensitizers for the ${ }^{1} D_{2}$ and ${ }^{1} G_{4}$ blue emitting thulium levels. The evolution of the population of the ${ }^{1} \mathrm{D}_{2},{ }^{1} \mathrm{G}_{4},{ }^{3} \mathrm{H}_{4}$, and ${ }^{3} \mathrm{~F}_{4}$ levels of $\mathrm{Tm}^{3+}$ and the ${ }^{2} \mathrm{~F}_{5 / 2}$ level of $\mathrm{Yb}^{3+}$ were investigated after short resonant excitation into these levels in YAG:Tm, Yb crystals. We observed upconversion fluorescence with a fast cooperative process followed by a slower step-by-step-process.
\end{abstract}

\section{Introduction}

The realization of laser emission from high energy levels of rare-earth ions in crystals is a difficult problem due to the lack of suitable pump sources. A way to overcome this difficulty would be to up-convert the infrared pump excitation by energy transfer between the activator ions or by using a sensitizer. Earlier experiments [1] indicated that $\mathrm{Yb}$ might be a good sensitizer for the blue emission of $\mathrm{Tm}$. However the interest in this sensitization was diminished by the lack of pump sources in the range 930-970 $\mathrm{nm}$ where $\mathrm{Yb}$ has a strong absorption band. The progress in powerful laser diodes emitting in this range renewed the interest in $\mathrm{Yb}$ sensitized systems. However the practical use of this up-conversion sensitization needs a deep knowledge of the dynamic and spectral properties of the system. We report here about the investigation of up-conversion of infrared radiation to the ${ }^{1} \mathrm{D}_{2}$ and the ${ }^{1} \mathrm{G}_{4}$ levels of Tm, $\mathrm{Yb}$ : YAG (Figure 1). The concentration of dopants varied from 1 to 3 at. $\%$ for $\mathrm{Tm}$ and from 5 to 20 at. $\%$ for $\mathrm{Yb}$.

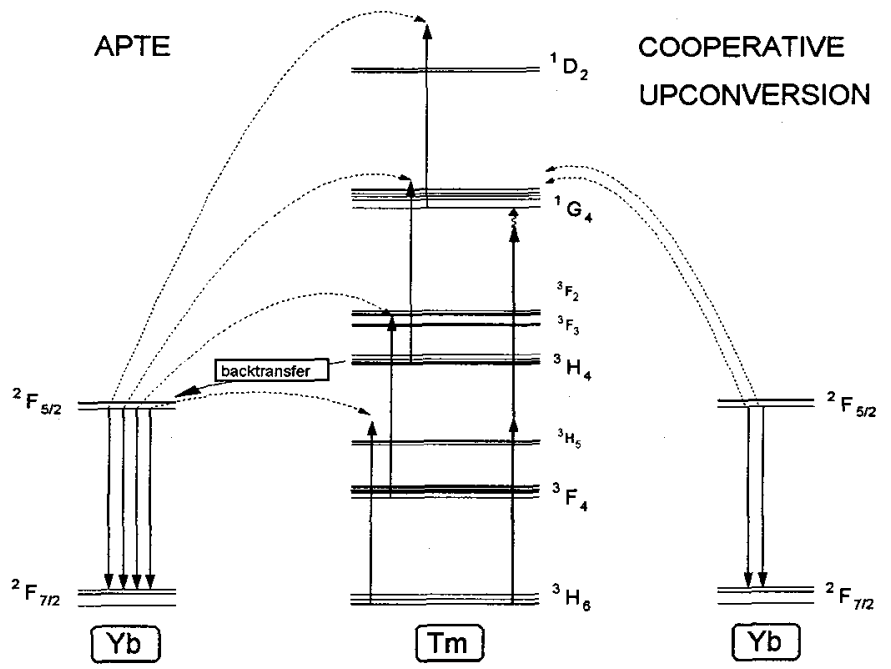

Fig. 1. Relevant energy levels and transfer processes in $\mathrm{Tm}$ and $\mathrm{Yb}$ in $\mathrm{YAG}$ 


\section{Experimental results}

We have investigated the temporal behaviour of luminescence from various levels of $\operatorname{Tm}\left({ }^{1} D_{2}\right.$, $1_{\mathrm{G}_{4}},{ }^{3} \mathrm{H}_{4}$, and ${ }^{3} \mathrm{~F}_{4}$, Figure 1) and from ${ }^{2} \mathrm{~F}_{5 / 2} \mathrm{Yb}$ when pumping into $\mathrm{Yb}$ or $\mathrm{Tm}$ levels (except of ${ }^{3} \mathrm{~F}_{4}$ ) with short dye, Ti: sapphire or OPO laser pulses.

Complex energy transfer takes place from $\mathrm{Yb}$ to $\mathrm{Tm}$ and vice versa:

- luminescence from all these levels of $\mathrm{Tm}$ is present when pumping into $\mathrm{Yb}$ with $940 \mathrm{~nm}$ radiation

- when pumping into ${ }^{3} \mathrm{H}_{4},{ }^{1} \mathrm{D}_{2}$ - and ${ }^{1} \mathrm{G}_{4}$-Tm emission occurs only in $\mathrm{Yb}$ codoped crystals

- Yb emission is excited when pumping the various levels of $\mathrm{Tm}$.

Under ${ }^{2} \mathrm{~F}_{5 / 2} \mathrm{Yb}$ pumping the luminescence decay of ${ }^{1} \mathrm{D}_{2},{ }^{1} \mathrm{G}_{4}$, and ${ }^{3} \mathrm{H}_{4}$ shows a peculiar behaviour, to our knowledge not reported previously. The temporal evolution of luminescence consists of a fast spike (where the time $t_{\max }$ corresponding to the maximum emission intensity $I_{\max }$, ranges from 4 to $25 \mu \mathrm{s}$ ) followed by a slow rise and decay (with $t_{\max }$ from 18 to 240 $\mu \mathrm{s})$. The values of $t_{\max }$ for the fast and the slow process and the ratio $I_{\max }$, fast $/ I_{\max }$, slow depend on the $\mathrm{Yb}$ and $\mathrm{Tm}$ concentrations and on the emitting level: at high concentrations the values of $t_{\max }$ are smaller for both processes and $I_{\max \text {,fast }}$ is larger than $I_{\max }$,slow (Figure 2).

Similar behaviour was observed for the ${ }^{1} \mathrm{D}_{2}$ and ${ }^{1} \mathrm{G}_{4} \mathrm{Tm}$ and ${ }^{2} \mathrm{~F}_{5 / 2} \mathrm{Yb}$ emission (Figure 3) when pumping into ${ }^{3} \mathrm{H}_{4}$ or for the ${ }^{2} \mathrm{~F}_{5 / 2} \mathrm{Yb}$ emission after excitation into ${ }^{1} \mathrm{D}_{2}$ or ${ }^{1} \mathrm{G}_{4}$. The fluorescence intensity from the investigated levels is nonlinear with the pumping intensity. The power dependencies are different for the various levels as well as for the fast and slow parts of the decay curve. In the sample with $1 \% \mathrm{Tm}$ and $10 \% \mathrm{Yb}$ the exponent of the ${ }^{1} \mathrm{G}_{4}$ fast emission process is about 2.2 , while for the slow process the exponents are 1.1 for ${ }^{3} \mathrm{~F}_{4}, 1.3$ for ${ }^{3} \mathrm{H}_{4}, 3.4$ for ${ }^{1} \mathrm{G}_{4}$, and 3.8 for ${ }^{1} \mathrm{D}_{2}$.

\section{Discussion}

The observed pump wavelength and concentration dependence implies that different up-conversion and depopulation processes are responsible for the fast and slow features observed in emission. The feed mechanism for the fast process is most likely a cooperative up-conversion excitation [2] into ${ }^{1} \mathrm{G}_{4}$ by which two excited $\mathrm{Yb}$ ions transfer simultaneously their excitation to a $\mathrm{Tm}$ ion. The depopulation of ${ }^{1} \mathrm{G}_{4}$ is also rather complicated and involves a three-ion process with an excitation of two $\mathrm{Yb}$ ions as well as an energy transfer within the Tm system. The dominant process seems to be the up-conversion $\left({ }^{1} G_{4},{ }^{1} G_{4}\right) \rightarrow\left({ }^{1} D_{2},{ }^{3} F_{3}\right)$, although the high efficiency of this process at relatively low $\mathrm{Tm}$ concentrations is surprising. The slow process could be due to a step-by-step (APTE) up-conversion [1] in which isolated Yb ions transfer successively their energy to various levels of a given $T \mathrm{~m}$ ion. A computer simulation based on a rate equation system gives a fairly well description of the observed behaviour with the parameters measured from the fluorescence decay curves of the various levels. In this analysis we used for the step-by-step $\mathrm{Yb}$ to $\mathrm{Tm}$ transfer an $\exp (-\beta \Delta \mathrm{E})$ dependence [3] for the various steps. Here, $\Delta \mathrm{E}$ describes the mismatch between $\mathrm{Yb}$ and $\mathrm{Tm}$ energy gaps for each step of transfer.

The large efficiency of the cooperative process seems surprising when regarding earlier estimations [4] which expect an efficiency several orders of magnitude lower than for the step-bystep process. This discrepancy might arise from the model of coupling: a strong short range interaction would lead to a dramatic increase of the rate of the cooperative processes. This correlates with other features of the process which suggest that the cooperative up-conversion takes place within nearest neighbours (n.n.) ensembles of two $\mathrm{Yb}$ ions and one $\mathrm{Tm}$ ion. 
$\operatorname{Tm}(1 \%), \mathrm{Yb}(5 \%)$ :YAG

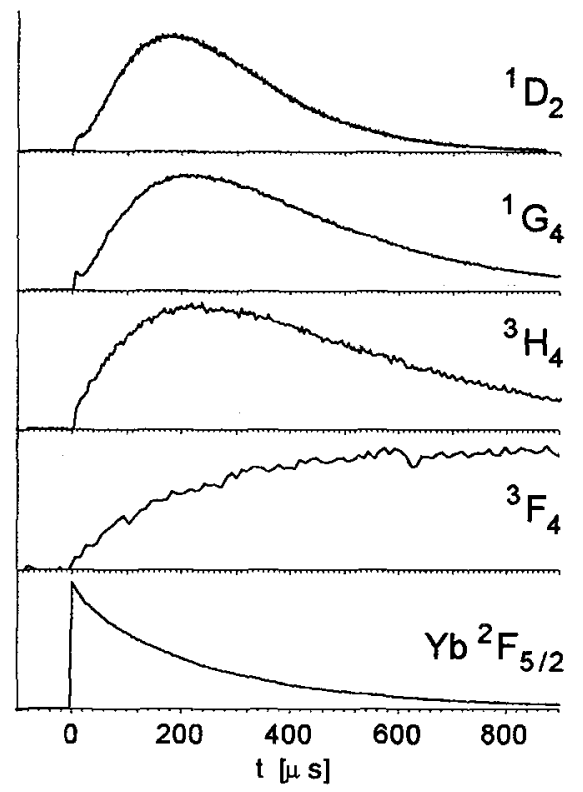

$\operatorname{Tm}(1 \%), \mathrm{Yb}(20 \%)$ :YAG

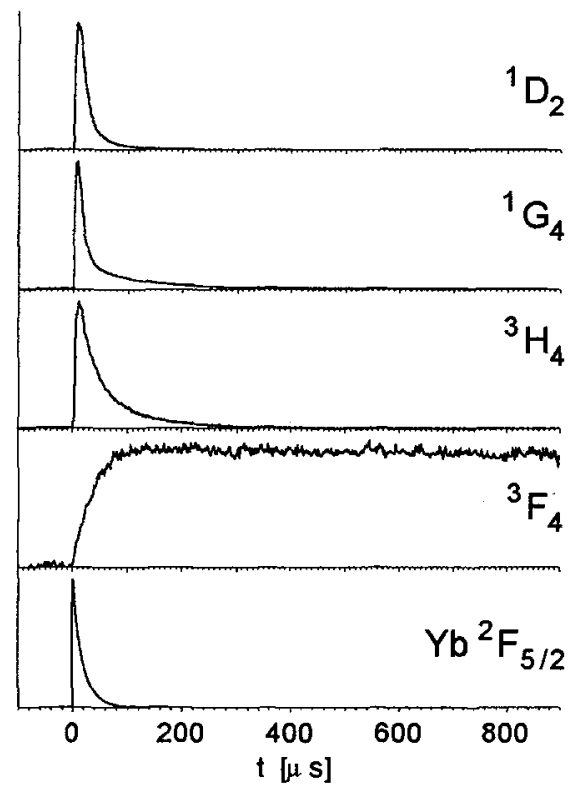

$\operatorname{Tm}(1 \%), Y b(10 \%): Y A G$

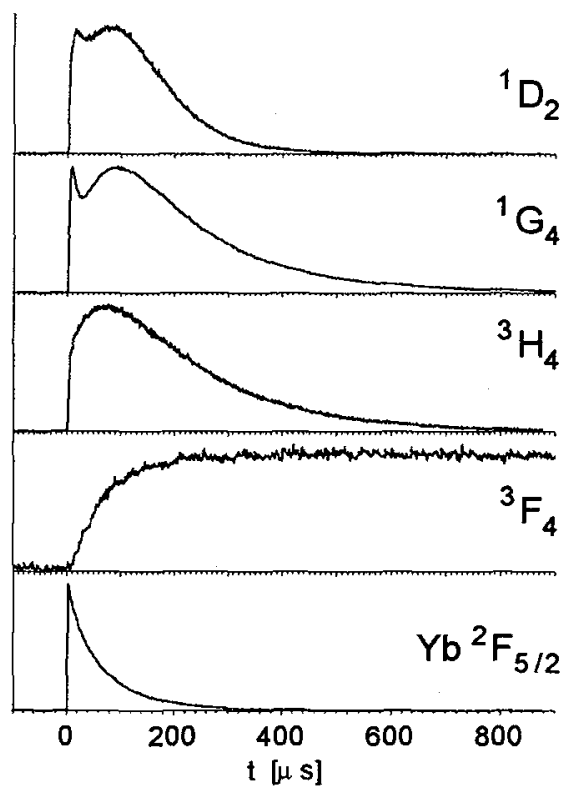

$\operatorname{Tm}(3 \%), Y b(10 \%): Y A G$

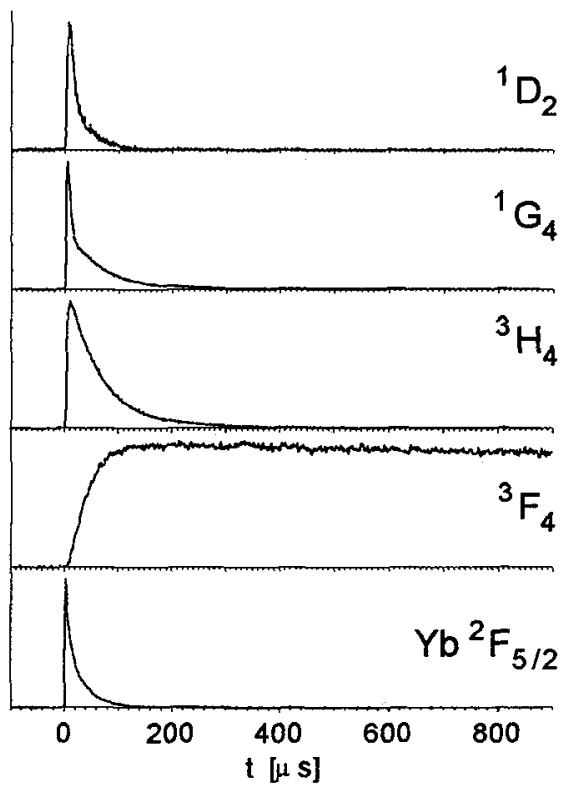

Fig. 2. Room temperature temporal behaviour of $\mathrm{Tm}{ }^{1} \mathrm{D}_{2},{ }^{1} \mathrm{G}_{4},{ }^{3} \mathrm{H}_{4},{ }^{3} \mathrm{~F}_{4}$ and $\mathrm{Yb}{ }^{2} \mathrm{~F}_{5 / 2}$ emission when pumping $\mathrm{Yb}^{2} \mathrm{~F}_{5 / 2}$ in different $\mathrm{YAG}$ :Tm, $\mathrm{Yb}$-crystals. The time scale is the same for all decays. 


\section{$\operatorname{Tm}(1 \%), \mathrm{Yb}(5 \%)$ :YAG}

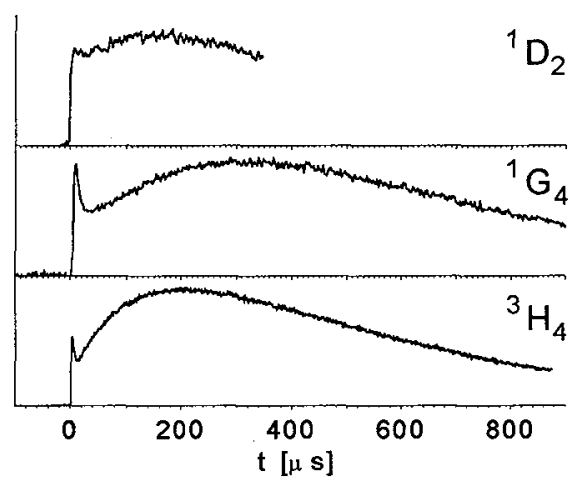

$\operatorname{Tm}(1 \%), \mathrm{Yb}(20 \%): Y A G$

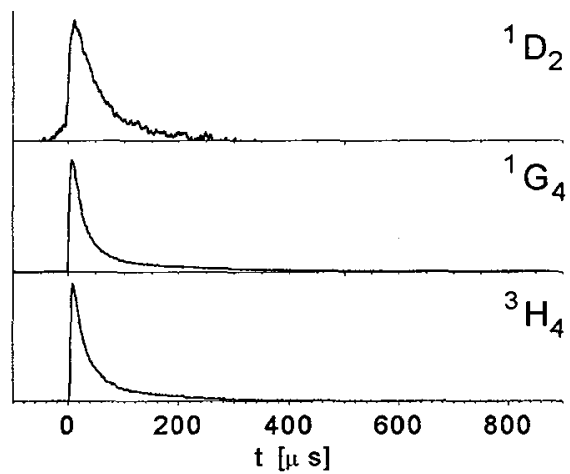

$\operatorname{Tm}(1 \%), Y b(10 \%): Y A G$

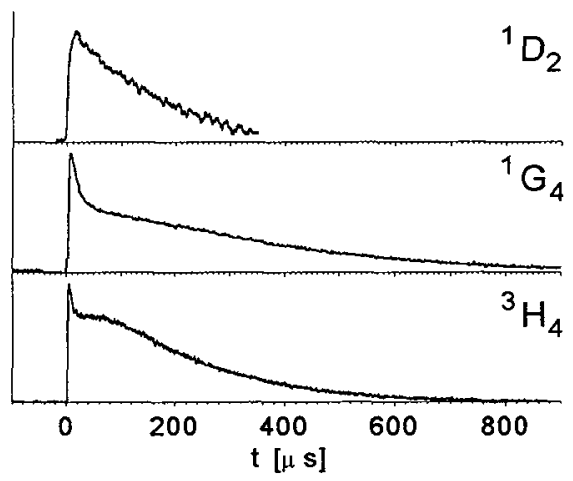

$\operatorname{Tm}(3 \%), Y b(10 \%): Y A G$

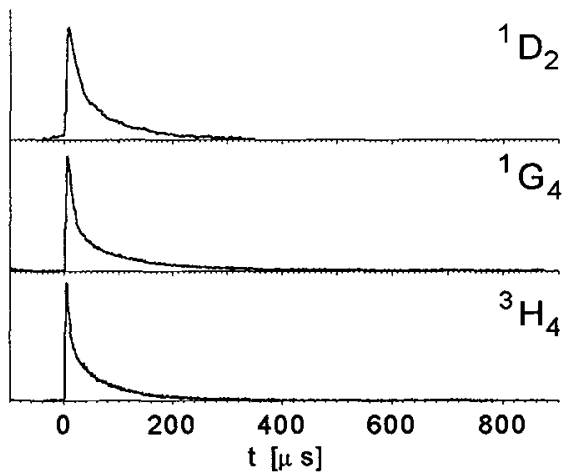

Fig. 3. Temporal behaviour of emission of ${ }^{2} \mathrm{~F}_{5 / 2} \mathrm{Yb}$ in $\mathrm{YAG}$ : $\mathrm{Tm}, \mathrm{Yb}$ when pumping in $\mathrm{Tm}$ ${ }^{1} D_{2},{ }^{1} G_{4}$, and ${ }^{3} H_{4}$. The other conditions are as in Figure 2 .

Also a rapid migration within $\mathrm{Yb}$ excited levels (i.e. high $\mathrm{Yb}$ concentration) would help to have the two n.n. Yb donor ions excited at the same moment. The possible presence of a superexchange interaction between the three ions of the ensemble, similar to that observed for several n.n. rare-earth ion pairs, could give consistency to our model.

\section{Conclusions}

The existence of the fast process in the population of the ${ }^{1} D_{2}$ or ${ }^{1} G_{4}$ level when pumping in $\mathrm{Yb}$, coupled with the slow rise of the population of ${ }^{3} \mathrm{~F}_{4}$ indicates inversion of population between these levels immediately after pumping, with potential for pulsed up-conversion laser emission.

[1] F. Auzel, C.R.Acad.Sci 262, 1016 (1966)

[2] V.V. Ovsyankin, P.P. Feofilov, Sov. Phys. JETP Lett. 4, 317 (1966)

[3] T. Miyakowa, D.L. Dexter, Phys. Rev. B1, 70 (1970)

[4] M. Stavola, D.L. Dexter, Phys. Rev. B20, 1867 (1979) 